\title{
Directives 2010 pour la réanimation
}

\section{Urs Klemmer}

Président Swiss Resuscitation Council

Médecin-chef adjoint REGA

\footnotetext{
* BLS: Basic Life Support; AED: Automated External Defibrillator
}

Correspondance:

Dr. med. Urs Klemmer case postale 1414 CH-8058 Zürich-Flughafen urs.klemmer@rega.ch

\section{Introduction}

Le 18 octobre 2010, l'American Heart Association (AHA) et l'European Resuscitation Council (ERC) ont publié simultanément de nouvelles directives pour la réanimation cardiopulmonaire. Celles-ci remplacent les directives de l'année 2005. Le but est de prendre en considération de nouvelles données probantes et de faciliter leur transmission de manière à pouvoir améliorer les chances de survie des patients. Les principaux changements concernent le domaine BLS, où des programmes d'instruction pour secouristes reposant sur une large base sont demandés.

L'AHA et l'ERC se fondent les deux sur les recommandations de l'ILCOR (International Liaison Committee on Resuscitation), qui réunit les organisations de réanimation nationales et internationales du monde entier. Les différences entre les recommandations de l'AHA et celles de l'ERC résident avant tout dans une interprétation différente des recommandations de l'ILCOR, là où le niveau de preuve scientifique laisse une certaine latitude.

Le SRC continue à viser une unité de doctrine dans le domaine des secouristes. Les mesures prises dans les premières minutes après un arrêt circulatoire sont absolument décisives pour la survie du patient. Celles-ci doivent être mises en œuvre par des personnes présentes par hasard et qui, dans la plupart des cas, ne sont pas qualifiées en médecine. La prise en charge de la période entre la survenue de l'arrêt circulatoire et l'arrivée des secours professionnels doit être améliorée en Suisse.

De ce fait, le SRC recommande des directives simples, claires et sans équivoque pour le domaine des secouristes et souhaite continuer à collaborer en vue d'établir, de façon interprofessionnelle et en incluant les organisations de secouristes et les écoles de sauvetage, des standards uniformes au niveau suisse. C'est pourquoi, l'algorithme bien connu du SRC et les recommandations de cours ont été adaptés aux nouvelles données et recommandations de l'ILCOR. Cela dit, l'établissement d'une unité de doctrine dans le domaine des secouristes ne réussira que si les divers intérêts particuliers sont subordonnés au but commun de former plus de personnes comme sauveteurs.

Dans le domaine des sauveteurs professionnels, le SRC soutient depuis des années les cours de réanimation éprouvés et couronnés de succès de l'AHA. Lors de l'introduction de ces cours, la Suisse faisait partie des pays pionniers. Toutefois, avec l'arrivée de médecins et d'ambulanciers étrangers, le nombre de diplômés des cours ERC a constamment augmenté dans notre pays. Vu l'absence de justifications techniques, il n'est plus opportun pour le SRC de préférer de façon unilatérale l'un des deux systèmes de cours. Pour les sauveteurs professionnels, le SRC soutiendra par conséquent, dès 2011, également les cours ERC en plus des cours AHA. Les sauveteurs professionnels pourront sans difficulté gérer les minimes différences sans qu'il en résulte de la confusion au quotidien.

Le SRC renonce donc encore et toujours à publier son propre algorithme pour les professionnels et recommande à la place aux spécialistes, pour les mesures ultérieures (ACLS/ALS et PALS/PLS), de suivre les cours standardisés et reconnus internationalement de l'AHA ou de l'ERC.

\section{Les changements les plus importants en 2010 pour les secouristes (Basic Life Support)}

L'algorithme BLS AED* a encore été simplifié par rapport à celui de 2005:

- Le point essentiel est le massage cardiaque, qui doit être commencé immédiatement après le constat de l'arrêt circulatoire.

- La fréquence des compressions lors du massage cardiaque est désormais d'au moins 100/min, la profondeur des compressions d'au moins $5 \mathrm{~cm}$ chez l'adulte ou d'au moins $1 / 3$ de l'épaisseur antéro-postérieure du thorax chez l'enfant.

- Comme jusque-là, il faut permettre une relaxation complète de la cage thoracique après chaque compression.

- Une séquence de réanimation commence toujours par le massage cardiaque.

- Eviter si possible toute interruption du massage cardiaque (continuer pendant que le défibrillateur se charge).

- Pour les personnes qui ont accompli une formation en Basic Life Support, il est recommandé de continuer à pratiquer tant le massage cardiaque que les insufflations (dans le rapport 30:2). Si la ventilation ne donne pas de résultat ou si aucune formation n'a été accomplie, les secouristes doivent s'en tenir au massage cardiaque. Les centrales d'urgence sanitaire doivent au besoin instruire sur le massage cardiaque les personnes présentes, par téléphone. 


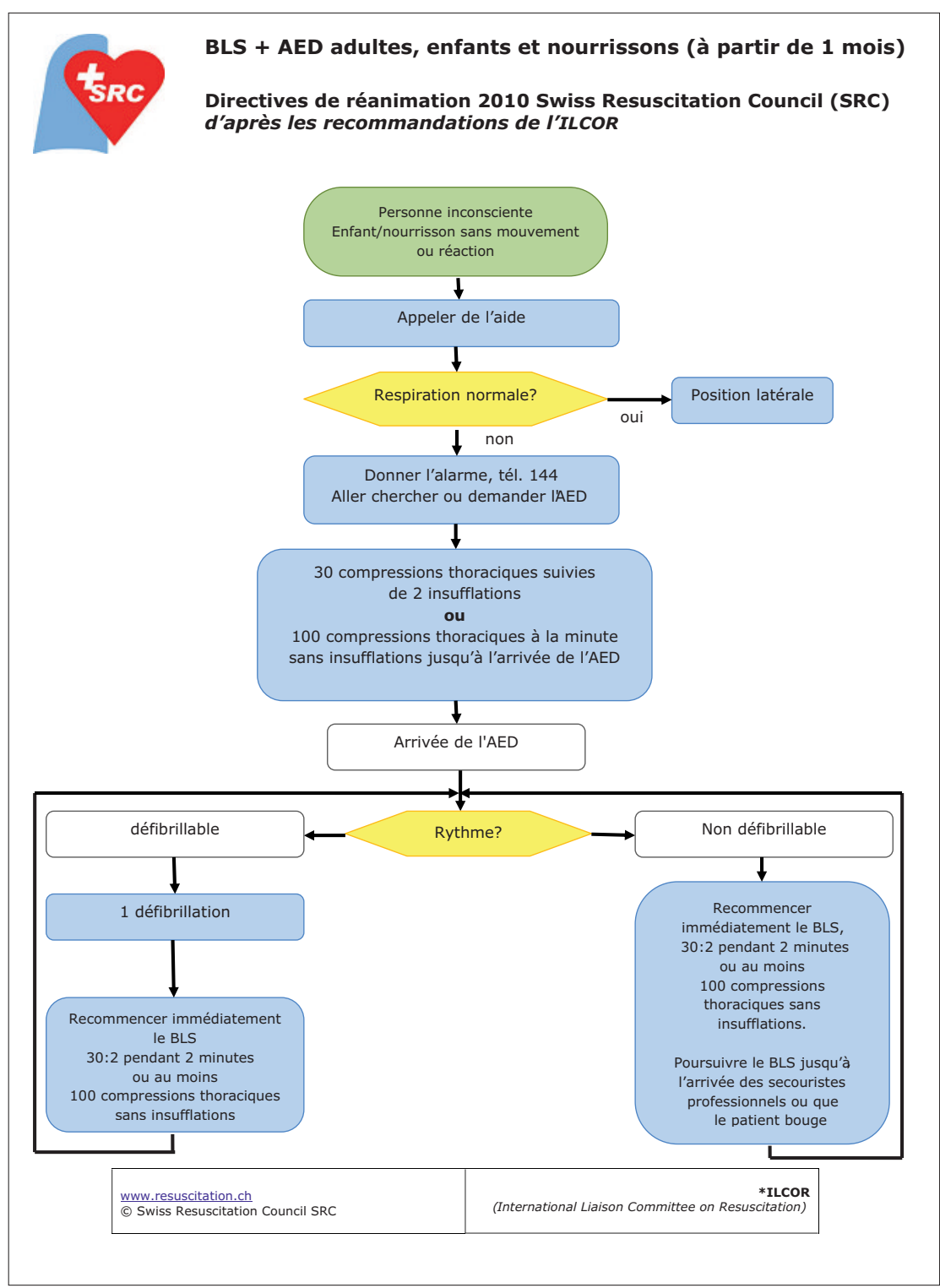

- L'évaluation de la respiration est plus brève (seulement une inspection visuelle du patient, plus de «voir-écouter-sentir»).

- Libération des voies respiratoires seulement si le secouriste maîtrise la technique nécessaire et si cela ne retarde pas le massage cardiaque.

- Les 2 insufflations initiales ne sont plus pratiquées.

- Même algorithme pour tous les patients dès l'âge de 1 mois.

- Dès qu'un AED est disponible, il doit être branché et allumé (plus de distinction entre arrêt circulatoire «observé» et «non observé»).

Dans le domaine des secouristes, l'accent est désormais mis sur le travail d'équipe et la possibilité d'exécution simultanée des mesures: un secouriste s'occupe de donner l'alarme alors qu'un deuxième commence déjà le massage cardiaque.

\section{Les changements les plus importants pour les secouristes professionnels en détail}

- Là aussi, le massage cardiaque, pratiqué avec le moins d'interruptions possible et une qualité optimale, a la priorité absolue.

- Seuls ceux qui sont entraînés peuvent effectuer des mesures plus étendues telle qu'une intubation endotrachéale pour sécuriser les voies respiratoires. L'interruption du massage cardiaque doit être limitée au minimum.

- La concentration en $\mathrm{CO}_{2}$ de fin d'expiration permet de surveiller la qualité du massage cardiaque et de reconnaitre rapidement la reprise spontanée de la circulation. Par conséquent, la mesure de $\mathrm{CO}_{2}$ est recommandée comme mesure routinière.

- Il faut éviter une ventilation excessive.

- Il n'est plus recommandé de pratiquer une pression cricoïde durant la ventilation.

- Les patients chez lesquels on soupçonne un infarctus du myocarde ou une attaque cérébrale profitent d'un transfert immédiat sans ralentissements et détours vers un hôpital équipé pour de tels cas (laboratoire de cathéter ou unité cérébrovasculaire).

- Lors de PEA et d'asystolie, l'administration d'atropine n'est plus recommandée.

- Une fois la réanimation réussie, les patients doivent être traités selon un protocole standardisé (hypothermie, contrôle de la glycémie, stabilisation de la circulation, contrôle de l'oxygénation, etc.).

\section{Résumé}

Largement simplifiées, les nouvelles directives pour les secouristes visent à inciter les personnes présentes par hasard lors de la survenue d'un arrêt circulatoire à mettre en œuvre les premières mesures essentielles à la survie. Par ailleurs, on attend toujours des personnes bénéficiant d'une formation étendue et des intervenants médicalement qualifiés des mesures plus différenciées et des réflexions de diagnostic différentiel correspondant à leur degré de formation. Ainsi, la formation en Suisse peut être proposée sous une forme simplifiée et échelonnée pour les secouristes.

Nous sommes conscients qu'un tel algorithme simplifié ne tient pas compte de certaines situations et que tous les algorithmes donnent lieu à des discussions. Toutefois, dans l'intérêt de l'objectif majeur, à savoir sauver plus de vies, un algorithme aussi simple que possible mais unique est nécessaire et judicieux. Nous pouvons ainsi améliorer ensemble la formation de cercles plus larges de la population, renforcer la chaîne de survie et sauver plus de vies.

\section{Pour plus d'informations, consulter les liens suivants}

Swiss Resuscitation Council SRC: www.resuscitation.ch

ILCOR: www.ilcor.org/en/home/

AHA: www.heart.org/HEARTORG/

ERC: www.cprguidelines.eu/2010/

Swiss Resuscitation Council (SRC) 